\title{
BEDT-TTF Radical Salts: Organic Metals and Superconductors
}

\author{
D. Schweitzer* \\ 3. Physikalisches Institut der Universität Stuttgart, \\ Pfaffenwaldring 57, D-7000 Stuttgart 80, Fed.Rep. of Germany
}

\begin{abstract}
A review of the structural, electronic and superconducting properties of some BEDT-TTF-radical salts is given.
\end{abstract}

\section{Introduction}

After the first discovery of superconductivity in an organic metal - the radical salts of TMTSF (tetramethyltetraselenafulvalene) - under pressure $/ 1 /$ and ambient pressure $/ 2 /$ soon it was clear that in addition to the usual intrastack contacts between the donor molecules, intermolecular contacts between molecules in neighbouring stacks are important in such materials. These interstack contacts result in a less pronounced one-dimensional electronic behaviour leading to a stabilization of the metallic character down to low temperatures.

Therefore, SAITO et al./3/ had the idea that the somewhat nonplanar structure of BEDT-TTF (bis-ethylenedithiolotetrathiofulvalene) might amplify the interstack contacts in radical salts of this donor resulting in an even more isotropic electric behaviour. Electrochemically prepared crystals of (BEDT-TTF) ${ }_{2}{ }^{\circ}$ $\mathrm{ClO}_{4}$ (TCE) $0_{5}$ showed in fact the typical electrical behaviour of a quasi twodimensional organic metal /3, 4/ down to low temperatures, but did not become superconducting. In a similar salt (BEDT-TTF) ${ }_{4}\left(\mathrm{ReO}_{4}\right)_{2}$ the two-dimensionality is somewhat less marked but PARKIN et al. $15,64 \mathrm{did}^{4} \mathrm{find}$ a superconducting state near $2 \mathrm{~K}$ under an isotropic pressure of about 4 kbar, whereby the isotropic pressure was necessary to suppress a metal-insulator transition at $80 \mathrm{~K}$ and ambient pressure. This was an important result because for the first time in an organic sulphur donor system, superconductivity was observed. Nevertheless, the electrochemical preparation of this radical salt is not easy due to the fact that a large number of radical salts with different stoichiometries and crystal structures might grow simultaneously. For that reason we started to prepare radical salts of BEDT-TTF with $I_{3}$ counterions in THF-solutions /7-9/ leading to two radical salts with identical stoichiometries but different structures (see fig. 1), the so-called $\alpha$ - and B-(BEDT-TTF) ${ }_{2} I_{3}$ phases. Both types of crystals can be easily distinguished by eye because $\alpha$-phase crystals have usually a plate-like shape while B-phase crystals are canted rhombohedrons. In addition the room temperature ESR linewidth of the conduction electrons can be used to discriminate unequivocal between both salts ( 70 to 110 Gauss for $\alpha$-phase crystals and 20 to 25 Gauss for B-phase crystals depending on the direction of the crystals with respect to the magnetic field $/ 10,11 /$ ).

$\alpha-(B E D T-T T F)_{2} I_{3}$ crystals are two-dimensional organic metals with a nearly isotropic electrical conductivity $\left(\sigma_{300} \approx 60-250 \mathrm{~s} / \mathrm{cm}\right)$ within the ab-plane, a 1000 times smaller conductivity in the $c^{*}$-direction and a metal insulator transition at $135 \mathrm{~K} / 8,9 /$. B-(BEDT-TTF) ${ }_{2} I_{3}$ crystals are two-dimensional metals as

* on leave from Max-Planck-Institut für Med. Forschung, Abt. Molekulare Physik, Jahnstr. 29, 690C Heidelberg, Fed. Rep. Germany 

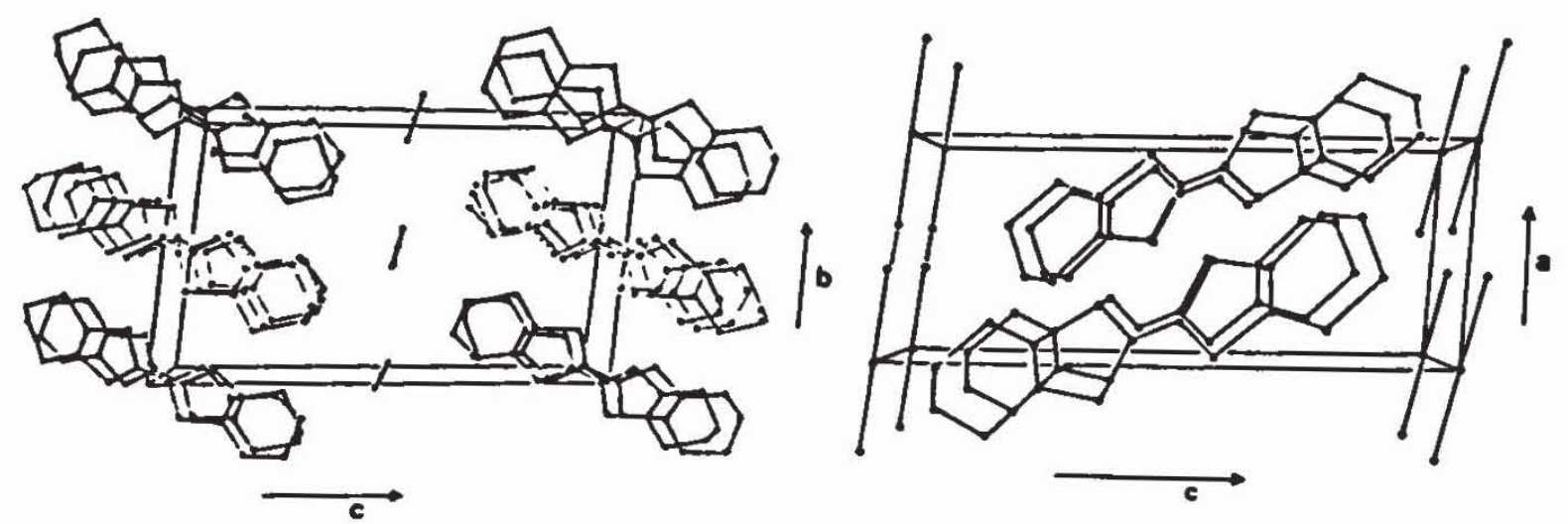

Figure 1 Stereoscopic projections of the structure of $\alpha$-(BEDT-TTF) ${ }_{2} I_{3}$ (left) and $B$-(BEDT-TTF) ${ }_{2} I_{3}$ (right) along the stacking axes.

well $\left(\sigma_{300} \approx 10-50 \mathrm{~S} / \mathrm{cm}\right.$ ) but stay metallic down to low temperature and - as was shown first by SCHEGOLEV et al./12/ - become superconducting at $1.3 \mathrm{~K}$ and ambient pressure.

In the following a review of the structural and physical properties of $\alpha$ and $B-(B E D T-T T F)_{2} I_{3}$ shall be given and the conditions will be described under which superconductivity at ambient pressure and $8 \mathrm{~K}$ in both types of crystals is observed.

$\underline{B-(B E D T-T T F)}_{2} \underline{I}_{3}$

The discovery of superconductivity in $B-(B E D T-T T F)_{2} I_{3}$ at $T_{0}=1.3 \mathrm{~K}$ and ambient pressure /12/ was confirmed by WILLIAMS et al. /13/ and Meissner-effect measurements $/ 14 /$ demonstrated the bulk property of the superconductivity in these crystals. A short time later it was found that under a pressure of $1.3 \mathrm{kbar}$ the superconducting transition in B-phase crystals can be raised to $7.5 \mathrm{~K} / 15,16 /$. After a particular pressure-temperature cycling procedure - pressurization up to $1.5 \mathrm{kbar}$ at room temperature and a release of the helium gas pressure at temperatures below $125 \mathrm{~K}$ - superconductivity at $T=8 \mathrm{~K}$ and ambient pressure was found $/ 17-19 /$. The observed sharp superconducting transition - as measured by the resistivity of the crystal - as well as the suppression of the superconducting state by a magnetic field along the $c^{*}$-axis at various temperatures is shown in fig. 2. The confirmation of bulk superconductivity at $8 \mathrm{~K}$ as well was obtained by Meissner-effect /20/ and ac-susceptibility measurements /18/. However, this specially prepared superconducting state at $8 \mathrm{~K}$ and ambient pressure

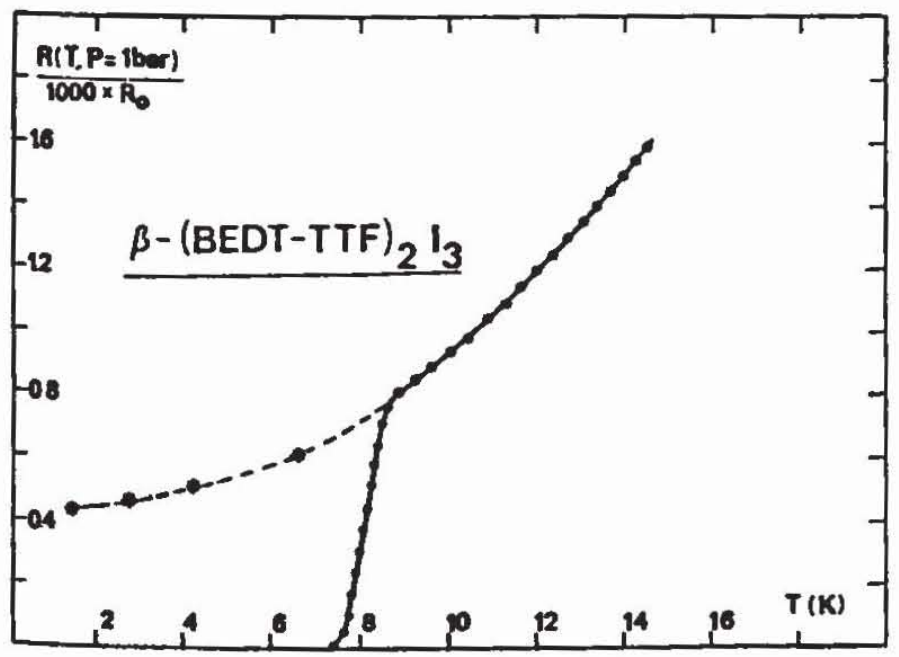

Figure 2

A superconducting transition at $8 \mathrm{~K}$ and ambient pressure in B-(BEDT-TTF $)_{2} I_{3}$ after a special pressure-temperature cycling procedure /17/ as well as the suppression by a magnetic field along the $c^{*}$-axis at various temperatures. 
is meta-stable because superconductivity can only be obtained as long as the crystal temperature does not exceed $125 \mathrm{~K}$. At this temperature a metal-metal phase transition was observed by thermopower measurements /10/. Recently it was shown that this meta-stable $8 \mathrm{~K}$ superconducting state can be obtained as well by an electronic excitation with laser light at temperatures below $125 \mathrm{~K} / 21 /$. In order to understand these somewhat surprising facts, the structural properties of the $B$-(BEDT-TTF) ${ }_{2} I_{3}$ crystals under the different conditions have to be discussed. At room temperature $B$-crystals are triclinic /22, 23/ $\quad(a=15.243$, $b=9.070, C=6.597 A ; a=109.73, B=95.56 ; \gamma=94.33^{\circ} ; V=848.9 A^{3}$ ) and the structure is very similar to the Bechgaard-salts. Below $195 \mathrm{~K}$ at ambient pressure an incommensurate structural modulation exists /24-26/ and the origin of this modulation is connected with an anion-cation interaction. Below $125 \mathrm{~K}$ a commensurate superstructure with a unit cell of about three times as large as at room temperature was observed /27/ ( $a=18.269, b=21.04, c=6.543 \mathrm{~A} ; \alpha=93.56, B=94.84$, $\left.\gamma=99.86^{\circ} ; V^{\prime}=2461 \mathrm{~A}^{3}\right)$. The basic structural change below $125 \mathrm{~K}$ with respect to the room temperature structure lies in a pronounced distortion of the triodide chains and especially in a change of the linear and symmetric $\mathrm{I}_{3}$-anions at room temperature into non-linear and asymmetric anions. This finding was confirmed by resonance Raman-investigations /21/. Therefore, it was assumed /27/ that the symmetric linear structure of the $\mathrm{I}_{3}$-anions at room temperature is stabilized down to low temperatures by the special temperature cycling procedure $/ 17,18$ / and this more symmetric structure results in the high $T_{\text {-superconducting tran- }}$ sition at $8 \mathrm{~K}$. In fact, this assumption was confirmed recently by neutron diffraction experiments $128,29 /$ and it was shown that no incommensurate modulated structure at $4.5 \mathrm{~K}$ exists, but furthermore, that the terminal ethylenegroups of the BEDT-TTF-molecules in the stacks are all ordered, in contrast to the low $T_{\text {-superconducting structure where only on one side the ethylene groups }}$ in the stácks are ordered, while on the other side they occupy the two possible conformations statistically /28, 29/.

It was shown /21/ that an electronic excitation of the B-phase crystals at temperatures below $125 \mathrm{~K}$ by laser light can induce - at least in a thin layer on the surface - the structural transformation from the low temperature $T_{0}=1.3 \mathrm{~K}$ structure into the more ordered and symmetric high temperature $T_{0}=8 \mathrm{~K}$ structure. This transformation is observed in the resonance Raman-spectrum by a disappearance of the splitting of the symmetric stretching mode of the $I_{3}$-anions with time at constant laser power (see fig. 3) or immediately at high light intensity $(\approx 50 \mathrm{~mW}) / 21 /$. Therefore, at temperatures between 1.5 and $8 \mathrm{~K}$ at least the surface of the crystal - in the moment it is not clear whether IRradiation might even switch the whole volume of the crystal - can be switched optically from the normal conducting into the superconducting state. But only those parts of the surface are switched which were irridiated by light (optical storage !). However, this transformation is again only stable as long as the crystal temperature does not exceed $125 \mathrm{~K} / 21 /$.

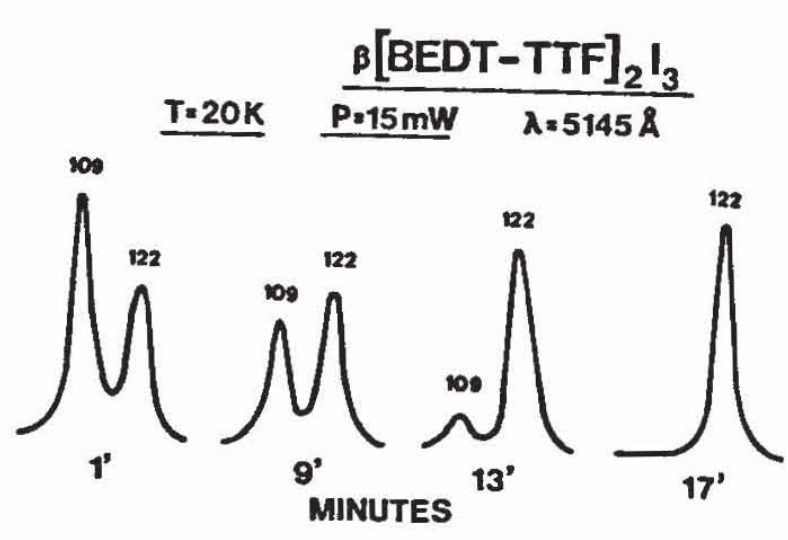

Figure 3

Structural transformation from the low into the high temperature superconducting phase in $\mathrm{B}-(\mathrm{BEDT}-\mathrm{TTF})_{2} \mathrm{I}_{3}$ by irradiation with light /21/ as observed by the change of the resonance Raman-spectrum of the symmetric stretching mode (109 and $122 \mathrm{~cm}^{-1}$ ) of the $\mathrm{I}_{3}$-anions with time at constant laser power (15 mW, at $5145 \AA$ ). 
$\alpha-$ and $\alpha+$-(BEDT-TTF) $2 I_{3}$

The unit cell of $\alpha$-(BEDT-TTF) ${ }_{2} I_{3}$ crystals (triclinic: $a=9.211, b=10.85$, $\mathrm{C}=17.488 \mathrm{~A} ; \alpha=96.95, B=97.97, v=90.75^{\circ}, V=1717 \mathrm{~A}^{3} / 9 /$ ) at room temperature is twice as large as the unit cell of the $B$-phase. In contrast to the $B$-phase crystals in $\alpha$-(BEDT-TTF) ${ }_{2} I_{3}$ two crystallographically different stacks occur and there exist large dihedral angles between the molecular planes (59.4 and $70.4^{\circ}$ ) of neighbouring donor molecules. The $\mathrm{I}_{3}$-anions are linear and all the terminal ethylene-groups of the BEDT-TTF-donor molecules in both crystallographically non-equivalent stacks are ordered. Under ambient pressure the $\alpha-(\text { BEDT-TTF })_{2} I_{3}$ undergoes a metal-insulator phase transition at $135 \mathrm{~K} / 7-9 /$, which can be suppressed by an isotropic pressure of $>12 \mathrm{kbar}$, but no sign of superconductivity could be observed down to $100 \mathrm{mK} / 30 /$. It was claimed /31/ that doping the $\alpha$-phase crystals with iodine results in a metallic state below the insulator phase transition at $135 \mathrm{~K}$ followed by a transition to superconductivity at around $3.2 \mathrm{~K}$. Similar results as in ref. /31/ for the resisitivity for such iodine-doped $\alpha$-crystals could be obtained by GOGU /32/ as well, but microwave conductivity $/ 33 /$ as well as ac-susceptibility measurements /32/ showed that this metallic state is not a bulk effect in these crystals.

Recently, BARAM et al./34/reported a structural transformation of $\alpha$-phase crystals into $B$-phase crystals by tempering the crystals at a temperature of $70-100^{\circ} \mathrm{C}$ for about 10 to 20 hours. This is a quite surprising result because of the essential differences in the structures of both phases but the structural transformation was confirmed by Weisenberg-pictures /34/. The most surprising fact after the structural transformation was that these crystals show a sharp decrease in resistivity at around $8 \mathrm{k}$, indicating a superconducting transition at this temperature, but proof by a simultanously applied magnetic field was not reported. Further it was not clear whether or not the eventual superconducting transition is a bulk effect in these tempered crystals.

Another open question was what the differences in the structures between the normal grown $B$-(BEDT-TTF) ${ }_{2} I_{3}$ and the tempered $\alpha_{t}$-(BEDT-TTF) ${ }_{2} I_{3}$ crystals are. (In the following tempered $\alpha$-phase crystals are called $\alpha_{t}-\left(\right.$ BEDT-TTF) $\left.{ }_{2} I_{3}\right)$. This is an important question since $B-(B E D T-T T F){ }_{2} I_{3}$ becomes superconducting under

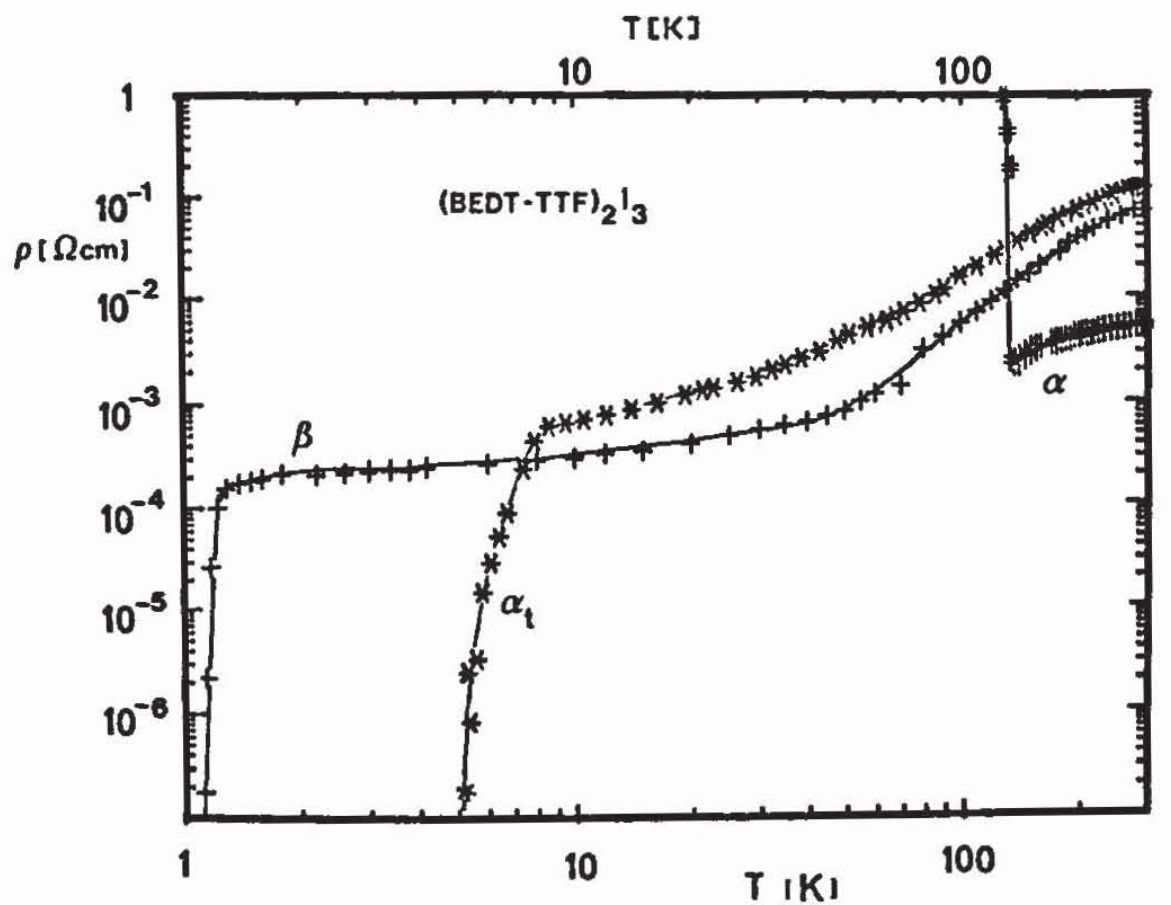

Figure 4 Resistivity versus temperature (logarithmic scales) for $\alpha-, \alpha_{t^{-}}$and $B-\left(\right.$ BEDT-TTF) ${ }_{2} \mathrm{I}_{3}$. 
normal conditions at $1.3 \mathrm{~K}$ while in $\alpha_{4}-\left(\right.$ BEDT-TTF ${ }_{2} \mathrm{I}_{3}$ this transition seems to be at around $8 \mathrm{~K}$, whereas the $\mathrm{B}$-(BEDT-TTF) ${ }_{2} \mathrm{I}_{3}$ shows superconductivity only after special treatment (temperature pressure cycling or electronic excitation by light).

A systematic study of the temperature-dependence of the resistivity (fig.4,5) and ac-susceptibility (fig. 6) both with and without applying a magnetic field as well as ESR- (fig. 7), NMR- (fig. 8), resonance Raman- (fig.9) and thermopower investigations on $\alpha_{t}-\left(\right.$ BEDT-TTF) ${ }_{2} I_{3} / 35 /$ has shown that bulk superconductivity at $8 \mathrm{~K}$ and ambient pressure exist in such $\alpha_{\text {-crystals. In contrast to }}$ the specially prepared $8 \mathrm{~K}$ meta-stable superconducting state in B-crystals here in $\alpha_{t}$-(BEDT-TTF) ${ }_{2} I_{3}$ the superconducting state is stable and entirely reproducable for several temperature cycles up to $380 \mathrm{~K}$.
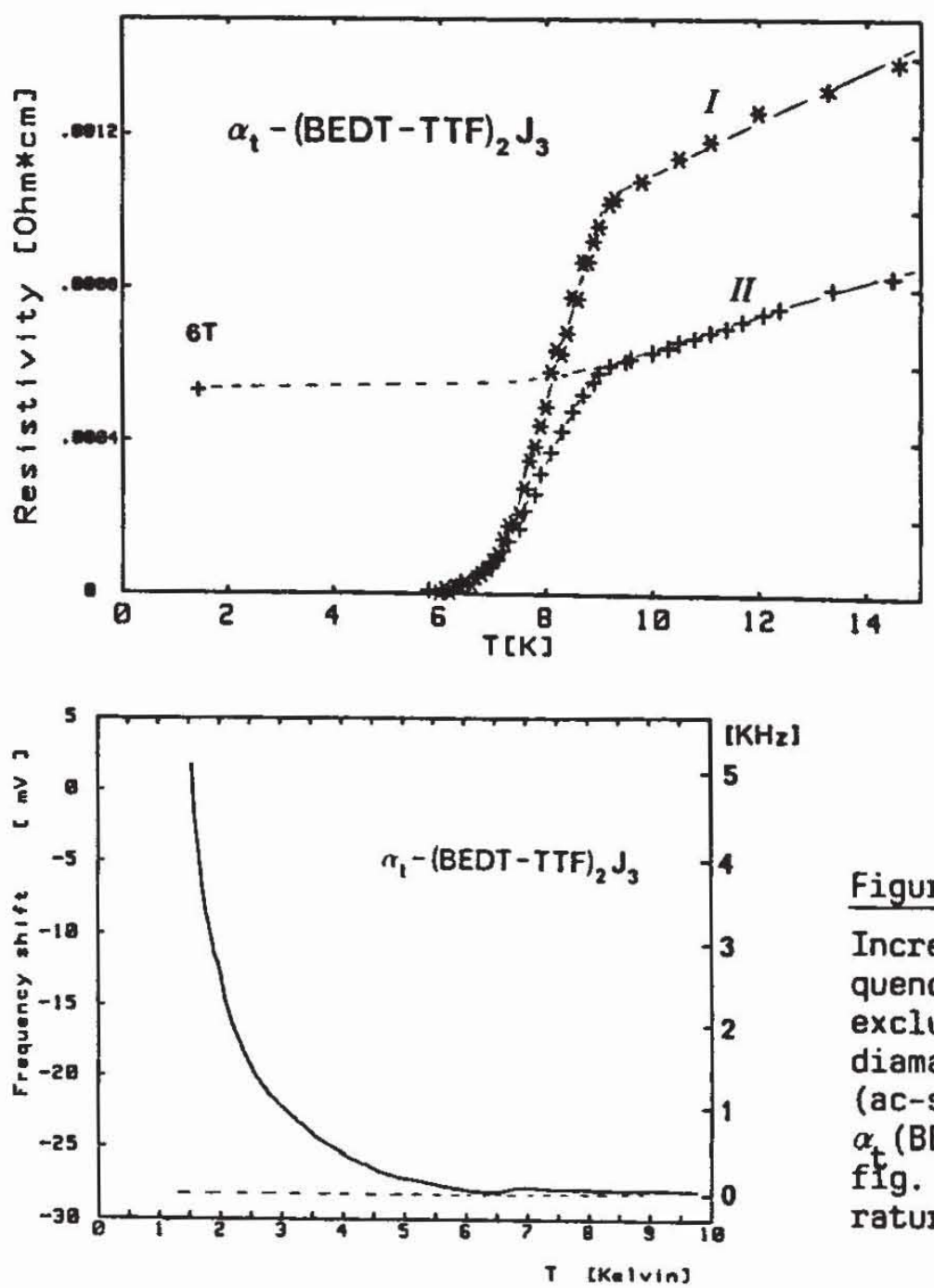

Figure 5

Resistivity of two $\alpha_{t}$-(BEDT-TTF) ${ }_{2} I_{3}$ crystals (I and II) in the temperature region between 0 and $15 \mathrm{~K}$ $/ 35 /$. The suppression of superconductivity in sample II by applying a magnetic field of $6 \mathrm{~T}$ at $1.3 \mathrm{~K}$ parallel to the $c^{\star}$-axis is shown as well.
Figure 6

Increase in resonance frequency of a LC-circuit due to exclusion of the rf-field by diamagnetic shielding currents (ac-susceptibility) in $\alpha_{t}$ (BEDT-TTF) ${ }_{2} I_{3}$ (sample II of flg. 5) by lowering the temperature $/ 35 /$.

${ }^{13} \mathrm{C}$-NMR solid state investigations /35/ ( $\mathrm{fig}$. 8) have shown that by tempering $\alpha$-crystals above $70^{\circ} \mathrm{C}$ for several days in air all crystals used in the experiment $(\approx 0.3 \mathrm{~g})$ were totally converted into the new $\alpha_{t}-(\text { BEDT-TTF })_{2} I_{3}-$
phase.

The ESR-linewidth measurements (fig. 7) as well as the resonance Raman-spectra (fig. 9) indicate the phase transition after tempering as well $/ 35 /$. The upper critical fields $H_{f 2}$ (fig. 10)/35/ are anisotropic but with 2.5 to $11 \mathrm{~T}$ (depending on the direction of the magnetic field with respect to the crystal axes) 

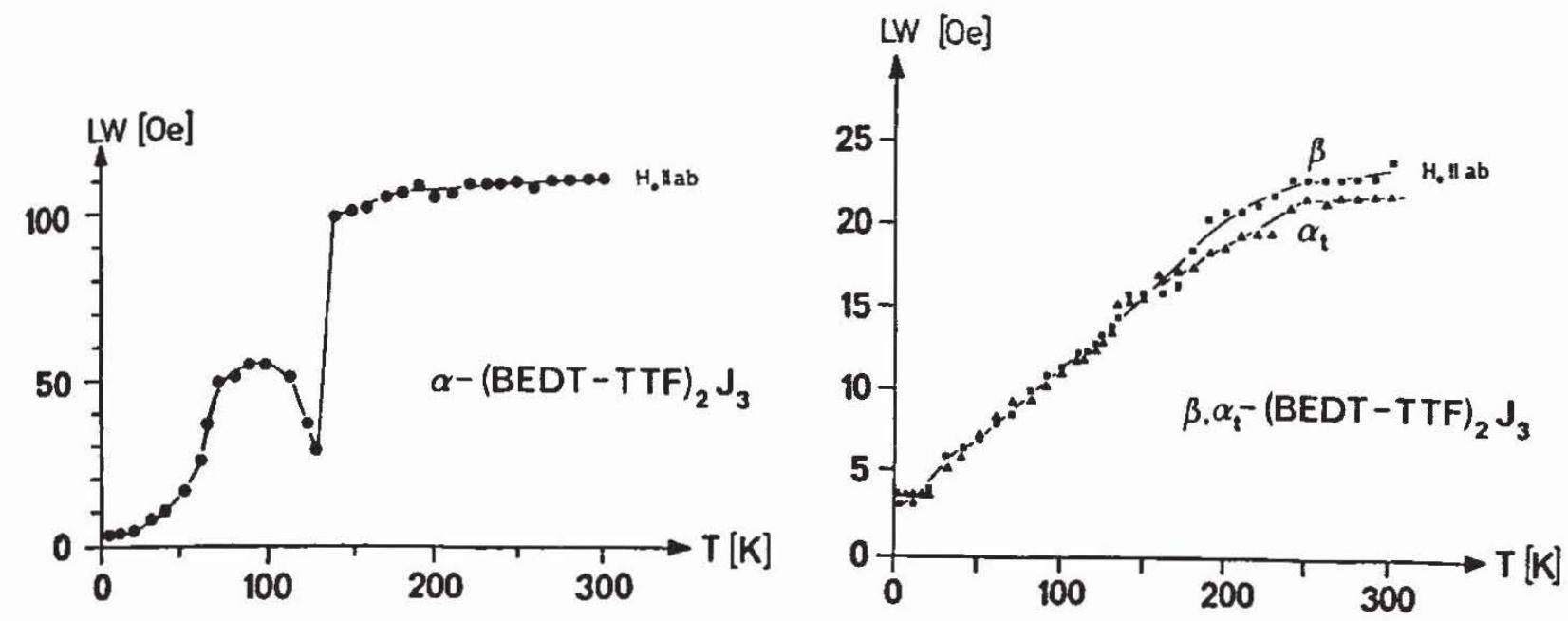

Figure 7 Temperature dependence of the ESR-linewidth for $\alpha-$ (BEDT-TTF) ${ }_{2} I_{3}$ (left) as well as for $B-(\bullet)$ and $\alpha_{t}-\left(\right.$ BEDT-TTF) ${ }_{2} I_{3}(\Delta)$ (right) $/ 35 /$.

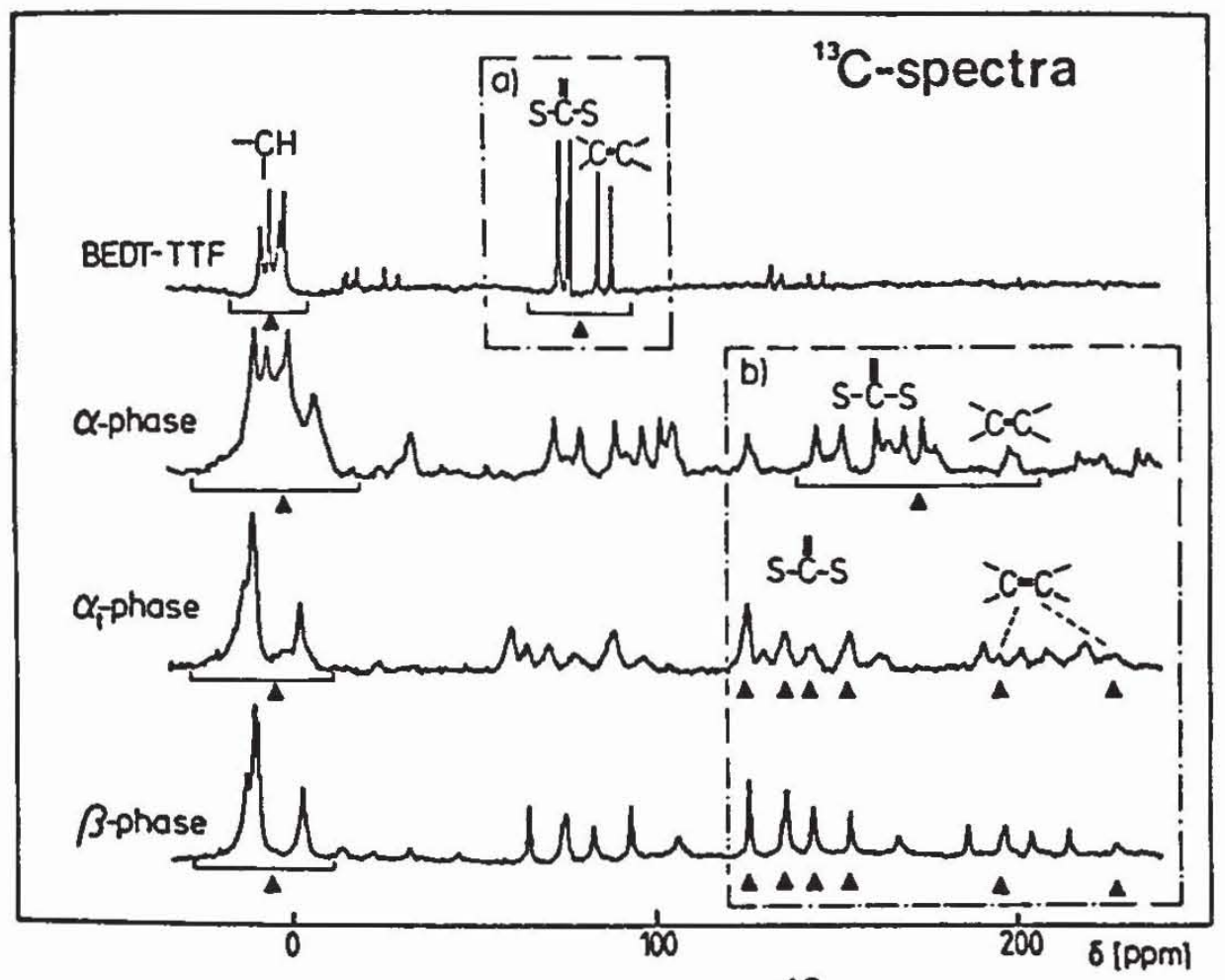

Figure 8 Magic angle spinning (MAS) ${ }^{13} \mathrm{C}$-NMR spectra at $300 \mathrm{~K}$ of BEDT-TTF and of the organic metals $\alpha-, B$ - and $\alpha_{-}$-(BEDT-TTF) ${ }_{2} I_{3}$. The center bands are marked by $\wedge$ in the framed parts as well as in the region of the ethylene groups ( $y=68 \mathrm{MHz}$, spinning frequency between 4-5 KHz)/35/.

relatively high. All the results from ref. $/ 34,35 /$ indicate that the $\alpha_{t}$-crystal structure at room temperature is identical with the one of the high $T_{\text {-super- }}$ conducting phase of $B$-crystals. This is probably due to the fact that ${ }^{c}$ in the $\alpha$-phase crystals at room temperature (and probably at $75^{\circ} \mathrm{C}$ as well) all terminal ethylene-groups of the BEDT-TTF donor molecules are ordered as in the

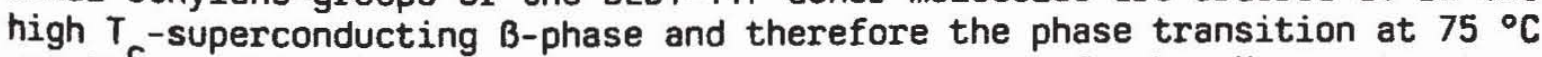
results in this structure: To clarify the situation, further $X$-ray structure investigations are necessary. 

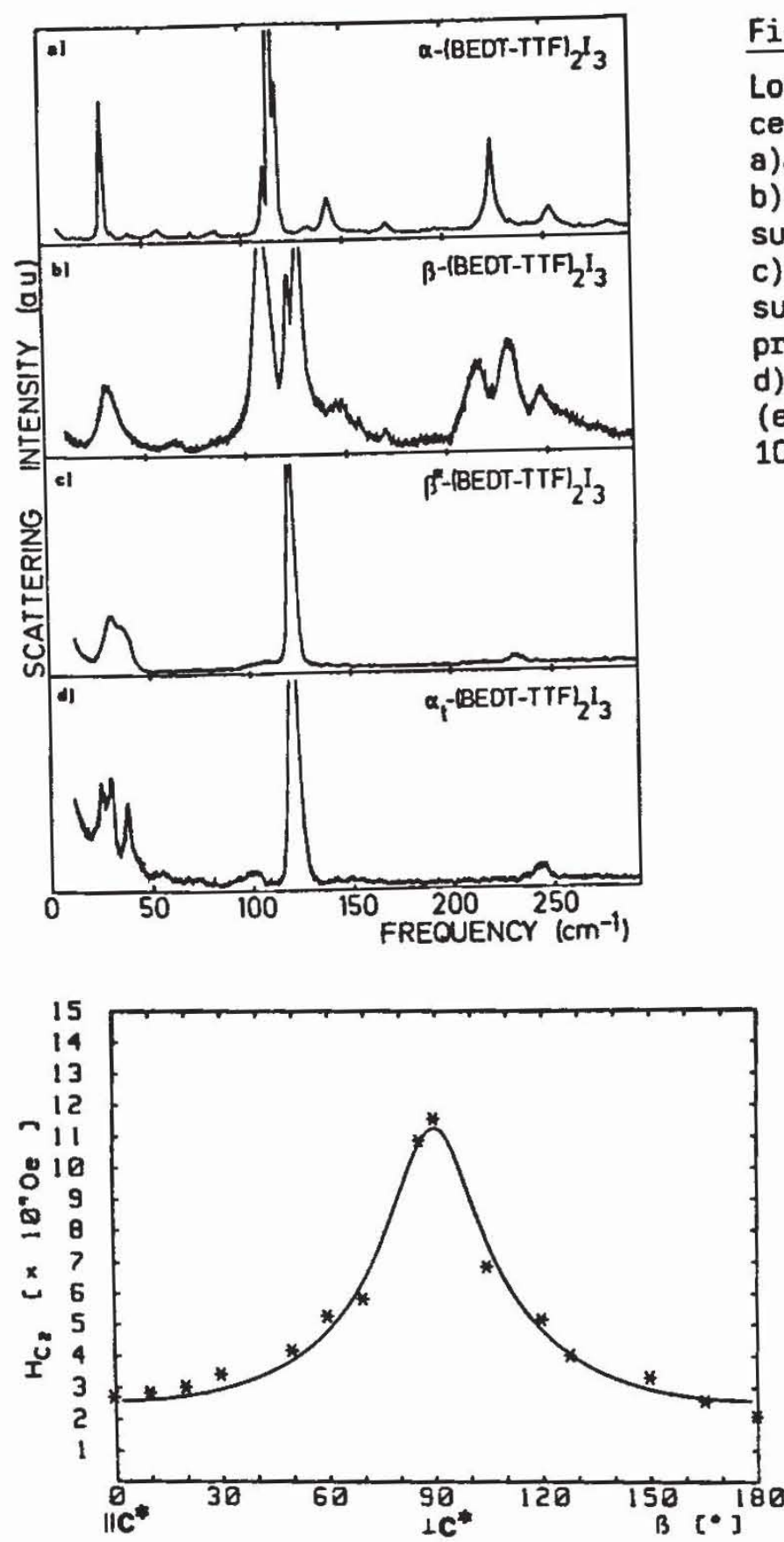

Figure 9

Low energetic parts of the resonance Raman-spectra at $2 \mathrm{~K}$ of

a) $\alpha$ - (BEDT-TTF) ${ }_{2} I_{3}$

b) B-(BEDT-TTF) ${ }_{2} I_{3}$ (low temperature superconducting $(1.3 \mathrm{~K})$ phase)

c) $B$ - (BEDT-TTF) ${ }_{2} I_{3}$ (high temperature superconducting $(8 \mathrm{~K})$ phase, as prepared by optical excitation/21/). d) $\alpha_{1}$ - (BEDT-TTF) ${ }_{2} \mathrm{I}_{3}$

(excitation wave length $4880 \mathrm{~A}$, $10 \mathrm{~mW}) / 35 /$.

\section{Figure 10}

Upper critical fields $\mathrm{H}_{\mathrm{c} 2}$ at $\alpha_{t}$-(BEDT-TTF) ${ }_{2} I_{3}$ as evaluated by the mid-transition of the resistivity curve for several magnetic field directions/35/.

\section{BCS superconductivity}

An important question is whether or not the usual electron phonon coupling is responsible for the superconductivity in the radical salts of BEDT-TTF. Tunneling experiments in the normal metallic /36/ as well as in the superconducting state $/ 37,38 /$ were carried out. Tunneling experiments of HAWLEY et al. /37/ on B-(BEDT-TTF) ${ }_{2}$ IAUI-crystals (superconducting transition at ambient pressure at $4.5 \mathrm{~K} / 39,40 /$ ) did show a superconducting gap $\Delta_{\mathrm{T}}$ in the ab-plane about 5 times larger than the expected BCS-value. More recent tunneling experiments /38/ on B-(BEDT-TTF) ${ }_{2} I_{3}$ as well as on B-(BEDT-TTF) ${ }_{2}$ IAUI in the ab-plane as well show values of $\Delta$ only about $15 \%$ larger than the BCS weak coupling value and are in very good accordance with the conventional electron phonon theory of superconductivity. However, the superconducting gap might be quite anisotropic and in fact preliminary band calculations /41/ indicate that the Fermi-surface 
has several separate sheets, which obviously may possess different superconducting gaps. In such a situation the average gap may correspond to the BCS-value and the maximum value of the gap may be considerably higher /38/. Therefore more experimental data for tunneling contacts with different orientations in the ab-plane are needed.

\section{Conclusions}

Bulk superconductivity at $8 \mathrm{~K}$ and ambient pressure exists in B-(BEDT-TTF) ${ }_{2} \mathrm{I}_{3}$ and $\alpha_{5}$-(BEDT-TTF) ${ }_{2} I_{3}$ crystals. While in the $B$-crystals the superconducting state has to be prepared under special conditions (temperature pressure cycling procedure or electronic excitation) and is only meta-stable, in $\alpha_{t}$-(BEDT-TTF) ${ }_{2} I_{3}$ this superconducting state at $8 \mathrm{~K}$ is stable and entirely reproducable for many temperature cycles up to $380 \mathrm{~K}$.

\section{Acknowledgement}

I would like to thank all my colleagues and co-workers (see references) for very effective cooperation during all the investigations in recent years. My special thanks to Professors H.J. Keller, H. Endres, M. Weger and Dr. Swietlik for many helpful discussions and efficient teamwork in Heidelberg.

\section{Literature}

1. D. Jérome, A. Mazaud, M. Ribault and K. Bechgaard: J. Phys. Lett. 4, L 95 (1980)

2. K. Bechgaard, K. Caneiro, M. Olsen, F.B. Rasmussen and C.S. Jacobsen: Phys. Rev. Lett. 46, 852 (1981).

3. G. Saito, T. Enoki, K. Toriumi and H. Inokuchi: Solid State Comm. 42 , 557 (1982).

4. G. Saito, T. Enoki, H. Inokuchi and H. Kobayashi: Journal de Physique 44, C 3, 1215 (1983).

5. S.S.P. Parkin, E.M. Engler, R.R. Schumaker, R. Lagier, V.Y. Lee, J.C. Scott and R.L. Greene: Phys. Rev. Lett. 50, 270 (1983).

6. S.S.P. Parkin, E.M. Engler, R.R. Schumaker, R. Lagier, V.Y. Lee, J. Voiron, K. Carneiro, J.C. Scott and R.L. Greene: Journal des Physique 44, C 3, 791 (1983).

7. D. Schweitzer, I. Hennig, K. Bender, K. Dietz, H. Endres and H.J.Keller: In Verhandlungen d. Deutschen Physikalischen Gesellschaft, Physikertagung Münster, März 1984, M0 32, p. 584 (1984).

8. K. Bender, K. Dietz, H. Endres, H.W. Helberg, I. Hennig, H.J. Keller, H.W. Schäfer and D. Schweitzer: Mol. Cryst. Liq. Cryst. 107, 45 (1984).

9. K. Bender, I. Hennig, D. Schweitzer, K. Dietz, H. Endres and H.J.Keller: Mol. Cryst. Liq. Cryst. 108, 359 (1984).

10. I. Hennig, K. Bender, D. Schweitzer, K. Dietz, H. Endres, H.J. Keller, A. Gleitz and H.J. Helberg: Mol. Cryst. Liq. Cryst. 119, 337 (1985).

11. R. Rothaemel, L. Forró, J.R. Cooper, J.S. Schilling, M. Weger, P.Bele, H. Brunner, D. Schweitzer and H.J. Keller: Phys. Rev. B34, 704 (1986).

12. E.B. Yagubskii, I.F. Schegolev, V.N. Laukhin, P.A. Kononovich, M.V. Kartsovnik, A.V. Zvarykina and L.I. Buravov: Sov. Phys. JETP Lett. 39, 12 (1984).

13. J.M.Williams, T.J. Emge, H.H. Wang, M.A. Beno, P.T. Copps, L.N. Hall, K.D. Carlson and G.W. Crabtree: Inorg. Chem. 23, 2558 (1984).

14. H. Schwenk, C.P. Heidmann, F. Gross, E. Hess, K. Andres, D. Schweitzer and H.J. Keller: Phys. Rev. B31, 3138 (1985). 
15. K. Murata, M. Tokumoto, H. Anzai, H. Bando, G. Saito, K. Jajimura and T. Ishiguro: J. Phys. Soc. Japan 54, 1236 (1985).

16. V.N. Laukhin, E.E. Kostyuchenko, Yu.V. Sushko, I.F. Schegolev and E.B. Yagubskii: Soviet Physics JETP Lett. 41, 81 (1985).

17. F. Creuzet, G. Creuzet, D. Jérome, D. Schweitzer and H.J. Keller: J. Physique Lett. 46, L-1079 (1985).

18. F. Creuzet, D. Jérome, D. Schweitzer and H.J. Keller: Europhys.Lettr. 1,461 (1986).

19. $\bar{F}$. Creuzet, C. Bourbonnais, D. Jérome, D. Schweitzer and H.J. Keller: Europhys. Lett. 1, 467 (1986).

20. H. Veith, C.P. Heidmann, F. Gross, A. Lerf, K. Andres and D. Schweitzer: Solid State Commun. 56, 1015 (1985).

21. R. Swietlik, D. Schweitzer and H.J. Keller: submitted to Phys. Rev. B.

22. T. Mori, A. Kobayashi, Y. Sasaki, H. Kobayashi, G. Saito and H. Inokuchi: Chem. Lett. 957 (1984).

23. V.F. Kaminskii, T.G. Prokhorova, R.P. Shibaeva and E.B. Yagubskii: JETP Lett. 39, 17 (1984).

24. P.C.W. Leung, T.J. Emge, M.A. Beno, H.H. Wang and J.M. Williams: J.Am. Chem. Soc. 106, 7644 (1984).

25. T.J. Emge, P.C.W. Leung, M.A. Beno, A.J. Schultz, H.H. Wang, L.M. Sowa and J.M. Williams: Phys. Rev. B. 30, 6780 (1984).

26. P.C.W. Leung, T.J. Emge, M.A. Beno, H.H. Wang, J.M. Williams, V. Petricek and P. Coppens: J. Am. Chem. Soc. 107, 6184 (1985).

27. H. Endres, H.J. Keller, R. Swietlik, D. Schweitzer, K. Angermund and C. Krüger: Z. Naturforsch. 41a , 1319 (1986).

28. A.J. Schultz, M.A. Beno, H.H. Wang and J.M. Willaims: Phys.Rev. B33, 7823 (1986).

29. A.J. Schultz, H.H. Wang, J.M. Williams and A. Filhol: J.Am. Chem. Soc. 108,7853 (1986).

30. H. Schwenk, F. Gross, C.P. Heidmann, K. Andres, D. Schweitzer and H.J. Keller: Mol. Cryst. Liq. Cryst. 119, 329 (1985).

31. E.B. Yagubskii, I.F. Schegolev, V.N. Laukhin, R.R. Shibaeva, E.E. Kostyuchenko, A.G. Khomenko, Yu.V. Sushko, A.V. Zvarykina, Pis'ma Zh. Eksp. Theor. Fiz. 40, 387 (1984).

32. E. Gogu: private communication.

33. M. Przybylski, H.W. Helberg, D. Schweitzer and H.J. Keller: Synthetic Metals 19, 191 (1987).

34. G.0. Baram, L.I. Buravov, L.C. Degtariev, M.E. Kozlov, V.N. Laukhin, E.E. Laukhina, V.G. Orischenko, K.I. Pokhodnia, M.K. Scheinkmann, R.P. Shibaeva and E.B. Yagubskii, JETP Lett. 44, 293 (1986).

35. D. Schweitzer, P. Bele, H. Brunner, E. Gogu, U. Haeberlen, I. Hennig, T. Klutz, R. Swietlik and H.J. Keller: submitted to Zeitschr.f. Physik B.

36. A. Nowack, M. Weger, D. Schweitzer, H.J. Keller: Solid State Comm. 60, 199 (1986).

37. M.E. Hawley, K.E. Gray, B.D. Terris, H.H. Wang, K.D. Carlson, J.M. Williams: Phys. Rev. Lett. 57, 629 (1986).

38. A. Nowack, U. Poppe, M. Weger, D.Schweitzer and H. Schwenk: submitted to Zeitschr. f. Physik B.

39. H.H. Wang, M.W. Beno, U. Geiser, M.A. Firestone, K.S. Webb, L. Nunez, G.W. Crabtree, K.D. Carlson, J.M. Williams, L.J. Azevedo, J.F. Kwak, J.E. Schirber: Inorg. Chem. 24, 2466 (1985).

40. E. Amberger, H. Fuchs and K. Polborn: Angew. Chem. 97, 968 (1985).

41. J. Kübler, M. Weger, C.B. Sommers: submitted to Sol. State Comm. 